\title{
GENERATION OF DISPATCHING SOLUTIONS IN A ROLLING TIME HORIZON FRAMEWORK WITH THE AID OF TABU SEARCH
}

\author{
ULLRICH MARTIN, MARKUS TIDEMAN \& WEITING ZHAO \\ Institute of Railway and Transportation Engineering (IEV), University of Stuttgart, Germany
}

\begin{abstract}
To increase the robustness of railway operation, at the Institute of Railway and Transportation Engineering (IEV) of the University of Stuttgart, a proactive dispatching algorithm has been developed. At the beginning, the block sections of a railway network are classified automatically depending on their specific operational risk index. Therefore, numerous timetables are modified based on random disturbances generated in a Monte Carlo scheme, then the modified timetables are simulated in RailSys $^{\circledR}$ software and, finally, the resulting waiting times in the studied network are calculated to obtain an operational risk map. Considering this operational risk map, near-optimal dispatching solutions are generated within a rolling time horizon framework. To this, the investigation time span is divided into so-called prediction horizons $(\mathrm{PH})$, which, on their part, are divided into so-called dispatching intervals (DI). At the start of each DI the train runs are artificially prolonged according to the risk map. Then, it is checked for the duration of the current $\mathrm{PH}$ whether conflicts (overlap of blocking times of at least two different trains in a block section) occur or not. If the detected conflicts are slight, they are solved by retiming of the affected train runs. However, in case of significant conflicts, the train runs have to be reordered. This is achieved with the aid of Tabu Search. In this paper, the functionality of the proposed algorithm is not only described but also demonstrated according to a realistic reference example that is standardly used at the IEV for algorithm development. In this context, special emphasis is placed on several important dispatching-related parameters to determine their general influence on the dispatching process. In the light of the reference example, it is shown that the proposed dispatching algorithm has a sustainable impact on the operation quality, particularly in comparison to other dispatching principles.

Keywords: dispatching, rescheduling, rolling time horizon framework, Tabu Search, risk mapping, operational risk analysis.
\end{abstract}

\section{INTRODUCTION}

During railway operation, the occurrence of random operational disturbances like dwell time extensions, running time extensions, departure time extensions and entry delays can lead to a significantly lower operational quality, e.g. in the form of resulting delays. Hence, besides buffer times to avoid or reduce transmission of delays between following trains, recovery times are added already during timetabling to reduce the impacts of disturbances of a train itself on railway operation (e.g. Kroon et al. [1] and Lindfeldt [2]). Admittedly, those recovery times should not become too large because of two aspects: Firstly, in congested areas there is no or only a small margin for additional time reserves and secondly, the operating capacity would deteriorate. Furthermore, longer scheduled travel times would result [3]-[5].

For this reason, railway operators commonly use conventional dispatching algorithms to soften the remaining effect of the disturbances during railway operation (e.g. D'Ariano [6]). In contrast to conventional dispatching algorithms, the Institute of Railway and Transportation Engineering (IEV) at the University of Stuttgart developed a dispatching algorithm, which considers further potential disturbances [7]. Therefore, the proposed dispatching approach consists out of two main processes. The first one is the operational risk 
analysis (Section 2) that provides the basis for the second process, which is the generation of dispatching solutions based on the Tabu Search (Section 3). Subsequently, the functionality of the proposed algorithm is demonstrated according to a realistic reference example (Section 4). At this one, special emphasis is placed on several important dispatching-related parameters to determine their general influence on the dispatching process. The present paper closes with an outlook for future research activities (Section 5).

To demonstrate the mode of action of the proposed dispatching principle, RailSys ${ }^{\circledR}$ software is used in different parts. This software not only allows to create a detailed infrastructure model and corresponding timetables but also to simulate the consequential railway operation [8]. As a main result of such a simulation, the software generates a log file which provides, inter alia, information about resulting delays of the train runs at any operating point of the investigated network.

\section{OPERATIONAL RISK ANALYSIS}

As this paper focusses especially on the generation of dispatching solution and the influence of dispatching-related parameters within the scope of a case study, the operational risk analysis is only described roughly in the following to support a basic understanding. For a detailed description of the operational risk analysis that is performed in offline mode reference is made to Zhao [9] and Zhao et al. [10].

At the beginning of this process, an investigated railway network is divided into appropriate block sections e.g. as shown in Li and Martin [11]. According to a fitting probability distribution of random disturbances a set of disturbed timetables is generated automatically in a Monte Carlo scheme for every block section separately. By imposing these so-called disturbance scenarios one by one on the railway operation on the corresponding block section and by simulating this in RailSys ${ }^{\circledR}$ software, the respective total weighted waiting time of each disturbance scenario can be obtained. Accordingly, the average total weighted waiting time can be calculated specifically for every block section. This average total weighted waiting time represents the operational risk of a specific block section. In general it can be said that the higher the average total weighted waiting is, the worse is the negative influence of disturbances occurring on the corresponding block section for the whole railway network.

To resort on the findings of the operational risk analysis in the context of the second process, which is the generation of dispatching solutions, the block sections are ranked according to their operational risk. This classification remains valid as long as the basic timetable and the infrastructure do not change significantly.

\section{GENERATION OF DISPATCHING SOLUTIONS}

Whereas the operational risk analysis is performed in offline mode, the generation of dispatching solutions is executed in online mode, viz. during railway operation. As shown in Fig. 1, this process consists out of four tasks [3], [9], [12].

Inter alia inspired by Zhan et al. [13], in the first task, the investigated time span is divided into so-called prediction horizons $(\mathrm{PH})$ and dispatching intervals $(\mathrm{DI})$ to enable a rolling time horizon framework that ensures a sustainable impact on the operational quality. Even though the respective duration has to be set by the user, it must be ensured that the investigated time span is an integer multiple of the length of the prediction horizons and that a prediction horizon is an integer multiple of the length of the dispatching intervals in turn.

At the beginning of every new dispatching interval, a conflict detection is performed (second task) for the length of the corresponding prediction horizon. To this goal, the blocking times of the train runs are artificially prolonged according to the results of the 
operational risk analysis. By doing so, potential disturbances occurring on the corresponding block sections are implicitly considered, whereby the robustness of the dispatching solution is increased. Then it is checked whether the current timetable contains conflicts in the shape of two or more overlapping blocking time stairways. In case of no detected conflicts, the current timetable will be maintained for the railway operation or rather its simulation (fourth task) until the beginning of the next dispatching interval. In the opposite case, a dispatching solution is generated for the current dispatching interval (third task). Depending whether a user-defined limit value for the target function, e.g. total weighted waiting time, is exceeded or not, the conflicts are treated as slight conflicts or significant conflicts.

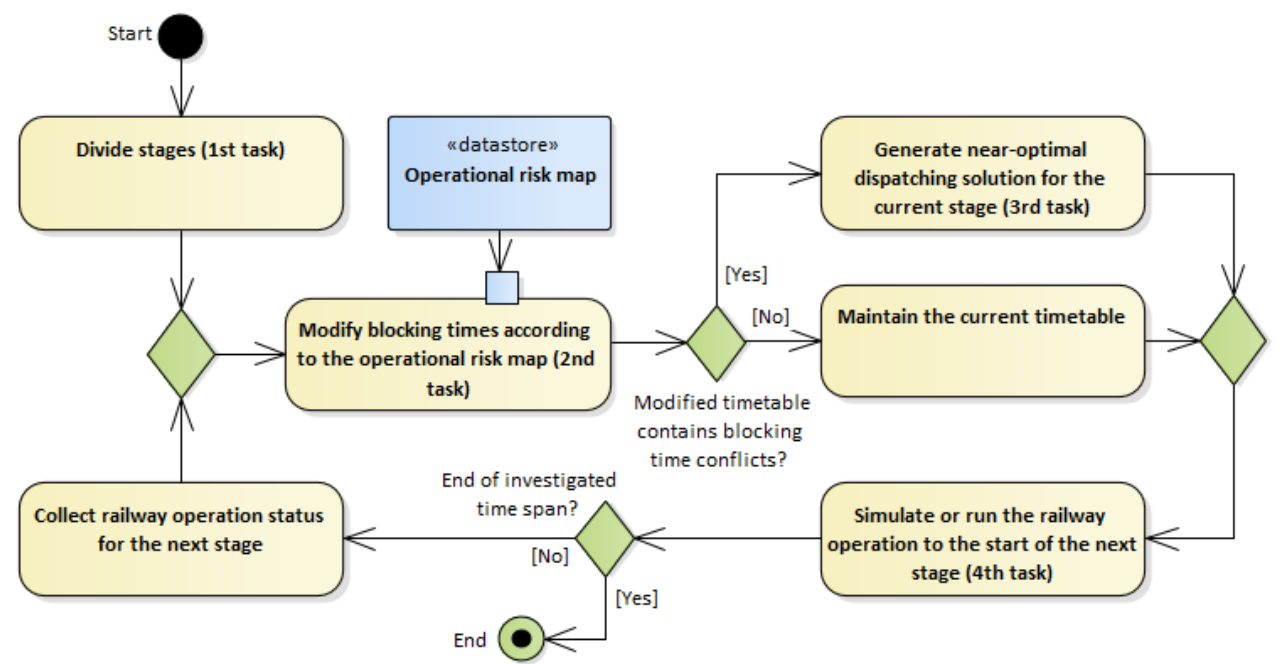

Figure 1: General workflow of the generation of dispatching solutions. (Source: Martin et al. [12].).

\subsection{Generation of dispatching solutions for slight conflicts}

Conflicts are considered as slight conflicts when the target indicator falls below a user-defined limit value. In order to save computation times and to minimize the deviation between the dispatched timetable and the original one, slight conflicts are solved only by retiming affected train runs. This means in particular that some train runs will be postponed, but the order of the train runs will not be changed [9].

\subsection{Generation of dispatching solutions for significant conflicts based on Tabu Search}

Whenever the value of the target indicator exceeds a user-defined limit value, the conflicts are solved by reordering the train runs. This means, that a better train sequence is gained. As a timetable usually consists out of a greater amount of trains, it would be very time consuming to calculate the value of the target function for every possible train order. This is why the proposed dispatching uses Tabu Search for reordering the train runs, which ensures a well-balanced compromise between calculation time and operational quality. Tabu Search method is a widely used in the field of transportation (e.g. Sterzik and Kopfer [14] and Corman et al. [15]). As depicted in Fig. 2, the user has to define aspiration and termination 
criteria, e.g. depending on the extension of the considered area or the expected number of trains to be scheduled. For example, as an aspiration criterion a value of the target function that is sufficiently good and as a termination criterion a maximum number of iterations could be set. The workflow of the reordering process starts with the current train order and an empty Tabu list. Then, all neighborhood train orders of the current train order are generated and saved as neighborhood timetables. This means just to switch the order of two trains in the train sequence. Afterwards, the value of the objective function for all these neighborhood timetables is calculated by simulating the railway operation in RailSys ${ }^{\circledR}$ software. Accordingly, the train order with the best objective function value is considered as the current train order and put on top of the Tabu list. As long as the termination criteria are not met, for this new current train order the neighborhood train orders are generated and their values of the objective function are calculated. If the best train order is not included in the Tabu list, the best train order is chosen as the new current train order and put on the top of the Tabu list. In case that the train order is included in the Tabu list, the sub-best train order is chosen as the new current train order and put on the top of the Tabu list, provided that the aspiration criteria are not fulfilled. Whenever the aspiration criteria are satisfied, the workflow interrupts and the current train order is treated as the dispatching solution. By using the Tabu list in the described manner, it can be avoided that the algorithm is stuck in a local minimum [9].

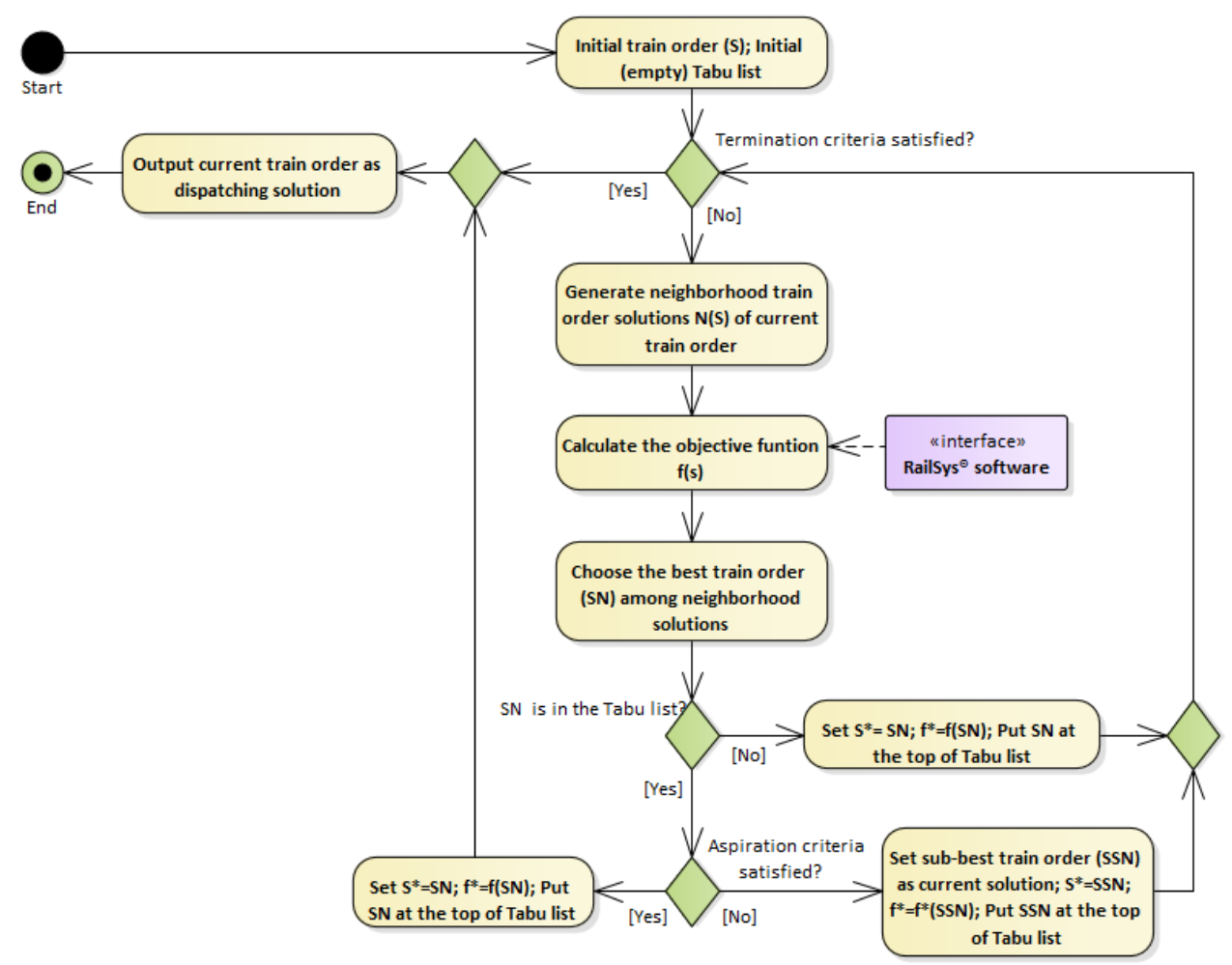

Figure 2: Workflow of the generation of dispatching solutions with the aid of Tabu Search. 


\section{CASE STUDY}

To demonstrate the impact of the proposed dispatching approach on operational quality, a realistic reference example is applied, which is standardly used at the Institute of Railway and Transportation Engineering (IEV) of the University of Stuttgart for algorithm development. This reference railway network is depicted in Fig. 3 and consists out of 43 kilometre track length with up to 72 train runs in six hours. "Technically, the operational risk analysis (first process) as well as the dispatching algorithm (second process) is developed in Microsoft Visual Studio 2015 environment with C\#. This code runs the simulations in RailSys ${ }^{\circledR}$ software automatically. Due to the large amount of disturbance scenarios that has to be imposed on every block section separately, the operational risk analysis has to be executed in an offline environment. For the reference scenario this process takes at least four hours (Fujitsu computer, Intel Core i5-4670 CPU @3.40 GHz, 8 GB RAM). After achieving the operational risk classification once, the classification will remain its validity in the future as long as there will not occur [significant] changes in infrastructure layout or operating

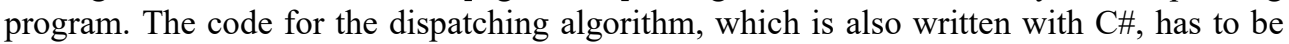
started manually by the user. With the objective of using this second process in online mode, the calculation of both conflict detection and conflict resolution takes about [only] 20 seconds for each stage" [3]. In the case study, the runs of regional passenger, freight transport and long-distance trains are artificially prolonged according to Section 2 (operational risk analysis) and to the conflict detection within Section 3 (generation of dispatching solutions) with the aid of additional recovery times representing entry delays, dwell time extensions, departure time extensions and/or running time extensions. By using the proposed approach instead of first come first serve-principle significant improvements regarding the resulting total weighted waiting times can be achieved within every DI as Table 1 indicates. Regarding the sums of the total weighted waiting times, the proposed dispatching approach ensures almost an improvement of a third compared to first come first serve-principle.

In this paper, special emphasis is placed on several important dispatching-related parameters to determine their general influence on the dispatching process. In this regard, a sensitivity analysis concerning, inter alia, the length of prediction horizon $(\mathrm{PH})$ and the length of dispatching intervals (DI) with respect to the investigated time span (TE) is conducted for the reference railway network described above. More precisely, the influence of different combinations of DI and $\mathrm{PH}$ by applying the dispatching solutions generated by the proposed approach on railway operation is explored in terms of the resulting total weighted waiting time, the average absolute retiming and the number of relative reordering.

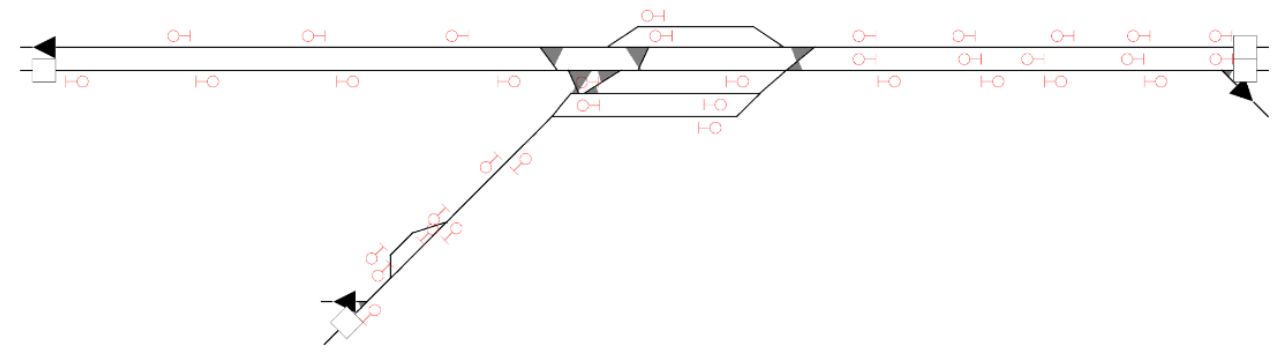

Figure 3: Track layout. (Source: Tideman et al. [3].). 
Table 1: Percentage improvement of the total weighted waiting time by using the proposed algorithm instead of first come first serve-principle within the case study.

\begin{tabular}{|c|c|c|}
\hline Dispatching interval (DI) & Time span & Improvement \\
\hline DI-0 & $0: 00-0: 30$ & $12 \%$ \\
\hline DI-1 & $0: 30-1: 00$ & $16 \%$ \\
\hline DI-2 & $1: 00-1: 30$ & $12 \%$ \\
\hline DI-3 & $1: 30-2: 00$ & $26 \%$ \\
\hline DI-4 & $2: 00-2: 30$ & $28 \%$ \\
\hline DI-5 & $2: 30-3: 00$ & $21 \%$ \\
\hline DI-6 & $3: 00-3: 30$ & $38 \%$ \\
\hline DI-7 & $3: 30-4: 00$ & $56 \%$ \\
\hline DI-8 & $4: 00-4: 30$ & $30 \%$ \\
\hline DI-9 & $4: 30-5: 00$ & $42 \%$ \\
\hline DI-10 & $5: 00-5: 30$ & $63 \%$ \\
\hline DI-11 & $5: 30-6: 00$ & $67 \%$ \\
\hline
\end{tabular}

In general, it is evident that the operational quality in the shape of total weighted waiting times gets better, the shorter DI and the longer PH is. This is due to two facts: First, the current railway operation status can be considered more effective by updating the dispatching solution very quickly (shorter DI). Second, dispatching solutions being generated by considering a greater time span (longer $\mathrm{PH}$ ) enable a long-lasting effect. However, it would be far too time consuming to run the dispatching algorithm in online mode for such combinations of PH and DI. Hence, the total weighted waiting time (TotalwWT) is calculated for the reference example for several combinations and the corresponding results are shown in Fig. 4. It should be noted that due to limited time budget during research work not any combination of DI and $\mathrm{PH}$ have been investigated, which is why the horizontal axis are non-linear scale in Figs 4-6.

Based on Fig. 4, one could tend to set PH as $50-60 \%$ of TE and DI as $10 \%$ of PH with regard to computational burden as this combination guarantees almost the same results as the best combination (PH 100\%, DI 5\%). In fact, to determine the most appropriate combination of PH and DI not only the total weighted waiting time but also the average absolute retiming (AAR) and the number of relative reordering (NRR) have to be considered. Analogous to Fig. 4, in Fig. 5 the resulting average absolute retiming and in Fig. 6 the number of relative reordering for different combinations of PH and DI are depicted, in each case averaged over all time stages for every combination. Both indicators are of great relevance in the context of railway dispatching as a dispatching solution should not only be good regarding the theoretical operational quality. Instead, the practical feasibility is also important so that for instance dispatchers and train drivers will not be overextended by rapidly changing timetables. While the averaged average absolute retiming does not variate too much, the number of relative reordering increases especially for prediction horizons being longer than approx. $40 \%$ of the investigated time span. On the contrary, the length of the dispatching interval has almost no influence on this indicator. Concluding the findings of this reference scenario, the length of PH should amount within $30 \%$ to $40 \%$ of TE and the length of DI should amount within $20 \%$ to $25 \%$ of $\mathrm{PH}$. 


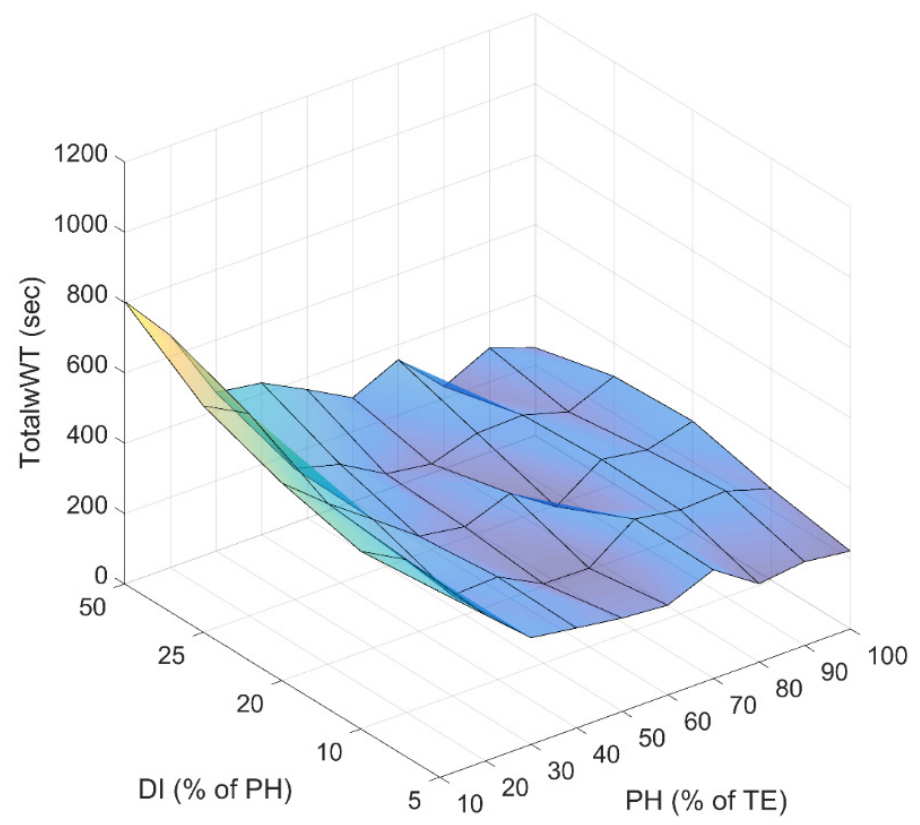

Figure 4: Variation of the total weighted waiting (TotalwWT) time against DI and PH for the reference example.

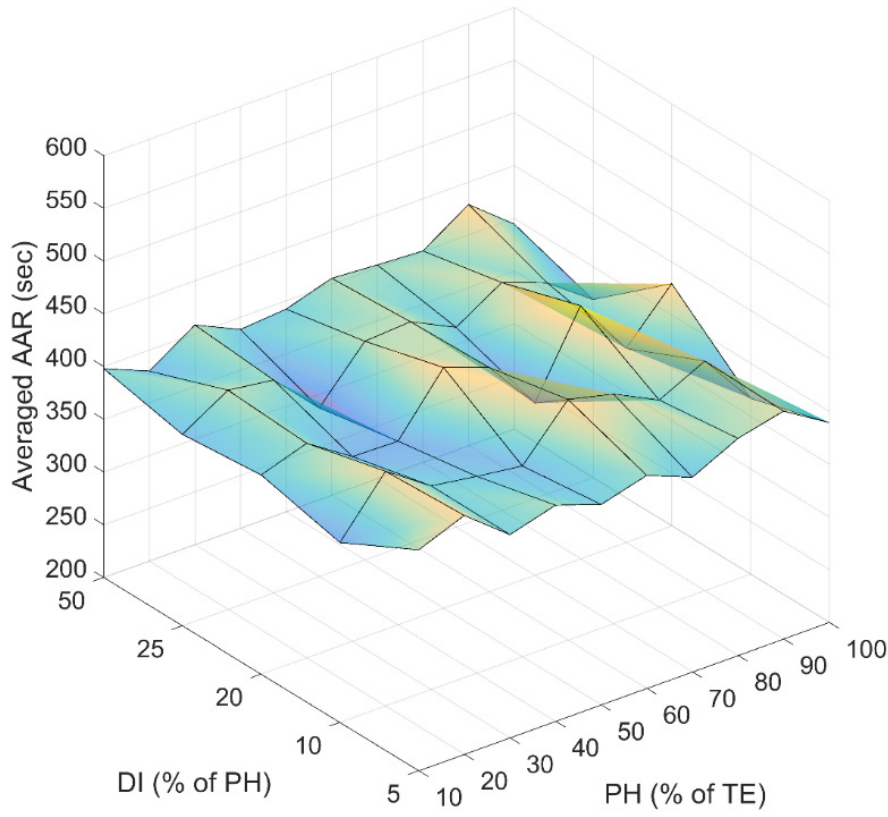

Figure 5: Variation of the averaged average absolute retiming (Averaged AAR) against DI and $\mathrm{PH}$ for the reference example. 


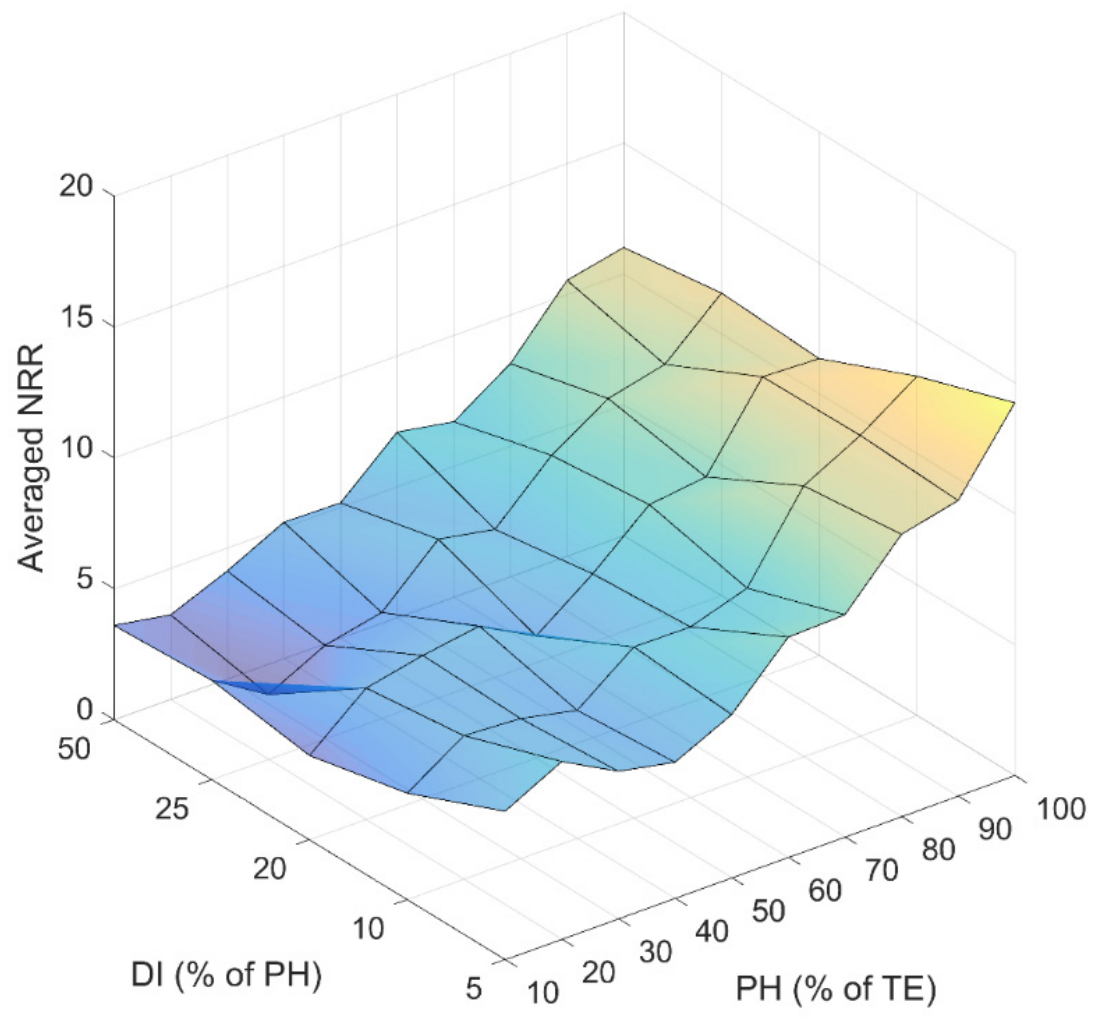

Figure 6: Variation of the number of relative reordering (Averaged NRR) against DI and $\mathrm{PH}$ for the reference example.

\section{CONCLUSION AND OUTLOOK}

The proposed dispatching algorithm offers numerous advantages, which are, for instance, its basic usability for every railway network and the improved operational quality compared with first come first serve-principle. In addition, the approach ensures practicable calculation times while generating robust dispatching solutions under consideration of future disturbances.

Based on the promising results, the proposed dispatching algorithm will be further developed. Suitable ideas and approaches are to transfer its operating principle to use cases belonging to other mode of transports [16], [17]. In this context, several adjustments have to be made. For instance, as in other transport modes no block sections exist a substitute is necessary to perform the operational risk analysis. In addition, the indicator representing operational risk might be replaced in cases where waiting times are not relevant. Furthermore, there are several use cases conceivable, in which the main objective of dispatching is not to better operational quality, but to reduce resource input or to use resources evenly.

Another promising intention is to implement methods of machine learning in the workflow of the operational risk analysis as well as the generation of dispatching solutions. In this field, the Institute of Railway and Transportation Engineering (IEV) at the University of Stuttgart has useful prior knowledge, especially originating from Tritschler et al. [18] and Bahamon et al. [19]. 


\section{ACKNOWLEDGEMENT}

We would like to thank the Deutsche Forschungsgemeinschaft (DFG) for funding that the research project Risk Oriented Dispatching of Railway Operation under the Consideration of Random Disturbances in Dynamic Circumstances (DICORD), on which the findings presented in this paper are based, made possible.

\section{REFERENCES}

[1] Kroon, L., Huisman, D. \& Maróti, G., Railway timetabling from an operations research perspective. Econometric Institute Report EI2007-22, pp. 1-25, 2007.

[2] Lindfeldt, A., Railway capacity analysis. Methods for simulation and evaluation of timetables, delays and infrastructure. Doctoral thesis, KTH Royal Institute of Technology: Stockholm, 2008.

[3] Tideman, M., Martin, U. \& Zhao, W., Proactive dispatching of railway operation. Proceedings of the 8th International Conference on Railway Operations Modelling and Analysis (ICROMA) - RailNorrköping, pp. 1605-1614, 2019.

[4] Cheng, Y., Hybrid simulation for resolving resource conflicts in train traffic rescheduling. Computers in Industry, 35(3), pp. 233-246, 1998.

[5] Espinosa-Aranda, J.L. \& García-Ródenas, R., A discrete event-based simulation model for real-time traffic management in railways. Journal of Intelligent Transportation Systems, 16(2), pp. 94-107, 2012.

[6] D'Ariano, A., Improving real-time train dispatching: models, algorithms and applications. Dissertation, TU Delft, 2008.

[7] Martin, U., Tideman, M. \& Zhao, W., Risk Oriented Dispatching of Railway Operation under the Consideration of Random Disturbances in Dynamic Circumstances (DICORD). DFG-project (MA 2326/22-1), in progress.

[8] Rail Management Consultants International GmbH. https://www.rmcon-int.de/railsysen/railsys-suite/. Accessed on: 24 Apr. 2020.

[9] Zhao, W., Hybrid Model for Proactive Dispatching of Railway Operation under the Consideration of Random Disturbances in Dynamic Circumstances, Books on Demand: Norderstedt, 2017.

[10] Zhao, W., Martin, U., Cui, Y. \& Liang, J., Operational risk analysis of block sections in the railway network. Journal of Rail Transport Planning \& Management, 7(4), pp. 245-262, 2017. https://doi.org/10.1016/j.jrtpm.2017.09.003

[11] Li, X. \& Martin, U., Ursachenbezogene Engpassbewertung in der Eisenbahnbetriebssimulation - DFG-Forschungsprojekt EPSUR. Proceedings of 25. Verkehrswissenschaftliche Tage Dresden, 2016.

[12] Martin, U., Tideman, M. \& Zhao, W., Generating robust dispatching solutions taking into account block sections' operational risk. Proceedings of the Fifth International Conference on Railway Technology: Research, Development and Maintenance (RAILWAYS 2020), submitted for publication.

[13] Zhan, S., Kroon, L., Zhao, J. \& Peng, J., A rolling horizon approach to the high speed train rescheduling problem in case of a partial segment blockage. Transportation Research Part E: Logistics and Transportation Review, 95, pp. 32-61, 2016. https://doi.org/10.1016/j.tre.2016.07.015

[14] Sterzik, S. \& Kopfer, H., A Tabu Search heuristic for the inland container transportation problem. Computers \& Operations Research, 40(4), pp. 953-962, 2013.

[15] Corman, F., D’Ariano, A., Pacciarelli, D. \& Pranzo, M., A Tabu Search algorithm for rerouting trains during rail operations. Transportation Research Part B: Methodological, 44(1), pp. 175-192,2010. 
[16] Tideman, M. \& Martin, U., Proaktive Disposition luftverkehrlicher Prozesse. Internationales Verkehrswesen, 70(4), pp. 60-63, 2018.

[17] Tideman, M., Martin, U. \& Zhao, W., Disposition von verkehrlichen Prozessen unter Einbeziehung von zufallsbedingten Unsicherheiten. Eisenbahntechnische Rundschau, 67(10), pp. 22-25, 2018.

[18] Tritschler, S., Cui, Y. \& Dobeschinsky, H., Störfallmanagement im ÖPNV. Der Nahverkehr, 23(10), pp. 14-18, 2005.

[19] Bahamon, B., Rapp, S., Rupp. C., Liu, J. \& Martin, U., Recognition of track defects through measured acceleration. Proceedings of the 7th International Conference on Euro Asia Civil Engineering Forum (EACEF), pp. 995-1010, 2019. 